\title{
Electromagnetic ion-cyclotron instability driven by combined loss- cone and temperature anisotropy distribution of suprathermal ions
}

\author{
HUA YuePing, WU DeJin ${ }^{*} \&$ WANG DeYu \\ Purple Mountain Observatory, Chinese Academy of Sciences, Nanjing 210008, China
}

Received June 29, 2010; accepted August 16, 2010

Electromagnetic ion cyclotron (EMIC) waves, particularly their generation and excitation mechanisms, have been a subject of wide interest because of their potential importance in ion acceleration and heating. In this work, the parameter-dependence of EMIC instabilities is investigated with a combined loss-cone and temperature anisotropy distribution for suprathermal ions. The calculation of the linear growth rate of EMIC waves with an arbitrary propagation angle is presented. The results show that the growth rates of EMIC waves propagating quasi-perpendicular to the ambient magnetic field increase as the loss-cone parameter $\alpha$ increases, whereas the growth rates of EMIC waves propagating quasi-parallel to the ambient magnetic field increase as the temperature anisotropy parameter $A_{T}$ increases. This indicates that the free energies associated with the loss-cone and temperature anisotropic distributions are primarily responsible for the excitation of the quasi-perpendicular and parallel propagating EMIC waves, respectively, and provides us with a more comprehensive understanding of excitation and generation mechanisms for EMIC waves in space plasmas.

acceleration of particles, plasmas, solar wind, waves

Citation: Hua Y P, Wu D J, Wang D Y. Electromagnetic ion-cyclotron instability driven by combined loss-cone and temperature anisotropy distribution of suprathermal ions. Chinese Sci Bull, 2011, 56: 257-262, doi: 10.1007/s11434-010-4267-0

Particle acceleration and electromagnetic fluctuation have proved to be important subjects in space exploration [1-4]. Electromagnetic ion cyclotron (EMIC) waves are often observed in various regions of the magnetosphere, ranging from a few to tens of RE (the Earth's radius). Their polarization properties, excitation mechanisms and wave-particle interactions have been extensively studied by a number of authors [5-11]. EMIC waves may have an important role in ion accelerating and heating. For instance, it is widely believed that the resonant absorption of EMIC waves is one of the main mechanisms for the acceleration and heating of high-speed solar wind streams [12-16]. In the Earth's magnetosphere, the heating of heavy ions has been linked to EMIC waves $[17,18]$. Also, EMIC waves are often used to explain ${ }^{3} \mathrm{He}$-rich events in impulsive flares in the solar corona $[19,20]$.

*Corresponding author (email: djwu@ pmo.ac.cn)
Observations within the Earth's magnetosheath have shown that the constituent ions often exhibit a temperature anisotropy $T_{\perp}>T_{/ /}$, where $T_{\perp}\left(T_{/ /}\right)$is the average over the particle distribution of $m_{i} v_{\perp}^{2} / 2\left(m_{i} v_{/ /}^{2}\right)$, with $m_{i}$ being the mass of the particle and $v_{\perp}\left(v_{/}\right)$being the component of the particle's velocity perpendicular (parallel) to the ambient magnetic field $B_{0}$ [21]. Statistical studies have demonstrated that there is an inverse correlation between the temperature anisotropy and the ion parallel $\beta_{i / /}=n_{i} T_{/ /} /\left(v_{0} B_{0}^{2} / 2\right) \quad$ [22]. Simulation studies of wave-particle scattering by the ion temperature anisotropy have yielded a similar inverse correlation. Furthermore, these simulation studies have shown that the wave-particle scattering is caused mainly by EMIC waves propagating parallel to $B_{0}$ that have been excited by the EMIC instability [23-27].

A study by Ludlow [28] showed that the temperature anisotropy can drive obliquely propagating EMIC waves. In 
this case, the growth rates of the EMIC waves do not depend strongly on the propagation angle $\phi$ for $\phi<0.5$, although for parallel propagation, the growth rates peak at $\phi=0$. Using a subtracted Maxwellian distribution, Denton and Hudson [29] argued that EMIC waves with larger propagation angles can be driven by loss-cone instability. Xue et al. [30] and Xiao et al. [31] further demonstrated that the Lorentzian distribution (also called the kappa distribution) can drive large-angle propagating EMIC waves as well.

However, in situ measurements by satellites in near-earth space plasmas have demonstrated that a large variety of microscopic velocity distributions of plasma particles exist that often cannot be modeled by a simple distribution function [32]. In the present paper, the growth rate of EMIC waves with the arbitrary propagation angle has been calculated using a combined loss-cone and temperature anisotropy distribution for suprathermal ions. These results support the previous work presented by the authors mentioned above, that the growth rates of quasi-perpendicular and quasi-parallel propagating EMIC waves are dominated by the loss-cone and the temperature anisotropy instabilities, respectively. This indicates that the free energy associated with the loss-cone distribution is mainly responsible for the excitation of the perpendicular propagating EMIC waves, while the free energy associated with the temperature anisotropy for the excitation is mainly responsible for the parallel propagating EMIC waves.

\section{Distribution function and growth rate}

Consider a plasma, which is uniformly magnetized by a constant magnetic field $B_{0}$ along the $z$ direction, consisting of a suprathermal ion population with mass $m_{i}$ density $n_{h}$, temperatures $\left(T_{\perp}>T_{/ /}\right)$. The background cold plasma has density $n_{0}>>n_{h}$ and temperature $T_{0}<<T_{/ /}<T_{\perp}$. The suprathermal ion population is assumed to have a combined velocity distribution function:

$$
f_{h}\left(v_{\perp}, v_{/ /}\right)=f_{h \perp}\left(v_{\perp}\right) f_{h / /}\left(v_{/ /}\right),
$$

where

$$
f_{h \perp}\left(v_{\perp}\right)=\frac{1}{2 \pi v_{T_{\perp}}^{2}} \frac{1}{\alpha !}\left(\frac{v_{\perp}^{2}}{2 v_{T_{\perp}}^{2}}\right)^{\alpha} \exp \left(\frac{v_{\perp}^{2}}{2 v_{T_{\perp}}^{2}}\right)
$$

and

$$
f_{h / /}\left(v_{/ /}\right)=\frac{1}{2 \pi v_{T_{/ /}}} \exp \left(\frac{v_{/ /}^{2}}{2 v_{T_{/ /}^{2}}^{2}}\right)
$$

are the perpendicular and parallel distribution functions, respectively; $\quad v_{T_{\perp}} \equiv \sqrt{T_{\perp} / m_{i}}$ and $v_{T_{/ /}} \equiv \sqrt{T_{/ /} / m_{i}}$ are the perpendicular and parallel thermal speeds of the suprathermal ions, respectively; $\alpha$ is the loss-cone index. For the case when the loss-cone index $\alpha=0$, the combined distribution function of eq. (1) is reduced to an anisotropic bi-Maxwellian distribution. The average parallel and perpendicular energies of the suprathermal ions in the combined distribution of eq. (1) are given by $\varepsilon_{/ /}=m_{i} v_{T_{/ /}}^{2} / 2$ and $\varepsilon_{\perp}=(\alpha+1) m_{i} v_{T_{\perp}}^{2}$, respectively. An effective temperature anisotropy can be represented by

$$
A_{T}=(1+\alpha) \frac{T_{\perp}}{T_{/ /}}-1=\frac{\varepsilon_{\perp}}{2 \varepsilon_{/ /}}-1 .
$$

It is well known that the general form of the dispersion equation can be obtained by letting the matrix determinant of the dispersion tensor $\left\{\Lambda_{i j}\right\}$ equal zero, that is $\Lambda \equiv\left\|\Lambda_{i j}\right\|=0$. Writing $\omega=\omega_{r}+i \gamma$ and invoking the approximation $\gamma \ll \omega_{r}$, the usual dispersion equation, which determines the wave frequency $\omega_{r}(\mathbb{k})$, can be obtained by taking the real part of the dispersion tensor $\lambda_{r}=\operatorname{Re}(\Lambda)$. The dispersion tensor is dominated by the ambient plasma in the approximation that $n_{h}<<n_{0}$. In the ambient cold plasma approximation, the dispersion equation can be written as [29]

$$
\Lambda_{r}=A N^{4}-B N^{2}+C=0
$$

with the coefficients

$$
\begin{aligned}
& A=P \cos ^{2} \phi+S \sin ^{2} \phi, \\
& B=(S-D) \sin ^{2} \phi+S P\left(1+\cos ^{2} \phi\right), \\
& C=P\left(S^{2}-D^{2}\right),
\end{aligned}
$$

where $N=c k / \omega_{r}$ is the refractive index, $c$ is the light speed, and $\phi$ is the propagation angle (i.e. the angle between the wave vector $\boldsymbol{k}$ and the $z$-axis). For low frequency waves, defined by $\omega_{r} \ll \omega_{p i}$ and $\Omega_{e}$ (where $\omega_{p i}$ is the ion plasma frequency and $\Omega_{e}$ is the electron gyrofrequency), the coefficients can be written as

$$
S=\frac{\omega_{p i}^{2}}{\Omega_{i}^{2}-\omega_{r}^{2}}, \quad D=\frac{\omega_{r}}{\Omega_{i}} \frac{\omega_{p i}^{2}}{\Omega_{i}^{2}-\omega_{r}^{2}}, \quad D=-\frac{m_{i}}{m_{e}} \frac{\omega_{p i}^{2}}{\omega_{r}^{2}} .
$$

The imaginary part of the dispersion tensor, $\Lambda_{i}=\operatorname{Im}(\Lambda)$, is dominated by contributions from the suprathermal ions and can be approximated by

$$
\begin{aligned}
\Lambda_{i}= & \lambda_{x x} \operatorname{Im}\left(\varepsilon_{x x}^{h}\right)+\lambda_{y y} \operatorname{Im}\left(\varepsilon_{y y}^{h}\right)+\lambda_{z z} \operatorname{Im}\left(\varepsilon_{z z}^{h}\right)+2 \lambda_{x y} \operatorname{Im}\left(\varepsilon_{x y}^{h}\right) \\
& +\lambda_{x z} \operatorname{Im}\left(\varepsilon_{x z}^{h}+\varepsilon_{z x}^{h}\right)+\lambda_{y z} \operatorname{Im}\left(\varepsilon_{y z}^{h}-\varepsilon_{z y}^{h}\right),
\end{aligned}
$$

where

$$
\begin{aligned}
& \lambda_{x x}=\left(N^{2}-S\right)\left(N^{2} \sin ^{2} \phi-P\right), \\
& \lambda_{y y}=-N^{2}\left(S \sin ^{2} \phi+P \cos ^{2} \phi\right)+P S, \\
& \lambda_{z z}=N^{4} \cos ^{2} \phi-N^{2}\left(S \sin ^{2} \phi+P \cos ^{2} \phi\right)+S^{2}-D^{2}, \\
& \lambda_{x z}=\lambda_{z x}=-N^{2}\left(N^{2}-S \sin ^{2} \phi\right) \sin \phi \cos \phi, \\
& \lambda_{x y}=D\left(P-N^{2} \sin ^{2} \phi\right), \\
& \lambda_{y z}=N^{2} D \sin \phi \cos \phi,
\end{aligned}
$$


are the elements of the adjoint of the dispersion tensor $\left\{\Lambda_{i j}\right\} \quad$ [33] and $\varepsilon_{i j}^{h}$ are the elements of the dielectric tensor of the suprathermal ions [34].

In general, for the case when the density of the suprathermal ions is low $\left(n_{h}<<n_{0}\right)$, the approximation $\gamma \ll \omega_{r}$ holds. The growth rate of the waves can then be calculated by [35]

$$
\gamma=-\frac{\Lambda_{i}\left(\omega_{r}, \mathbb{k}\right)}{\partial_{\omega_{r}} \Lambda_{i}\left(\omega_{r}, \mathbb{k}\right)},
$$

where $\partial_{\omega_{r}}$ denotes the partial derivative with respect to $\omega_{r}$. The growth rate, normalized by the ion gyrofrequency $\Omega_{i}$, can be obtained by

$$
\begin{aligned}
\frac{\gamma}{\Omega_{i}}= & \frac{n_{h}}{n_{0}} \frac{\omega_{p i}^{2}}{\omega_{r} \Omega_{i}} \frac{2 \pi^{2}}{G} \sum_{s=-\infty}^{+\infty} \int_{-\infty}^{+\infty} \mathrm{d} v_{/ /} \int_{0}^{+\infty} \mathrm{d} v_{\perp} \delta\left(\omega_{r}-s \Omega_{i}-k_{/ /} v_{/ /}\right) v_{\perp}^{2} \\
& \times\left[\left(\frac{s}{b} \lambda_{x y}-\frac{v_{/ /}}{v_{\perp}} \lambda_{y z}\right) J_{s}(b)-\lambda_{y y} J_{s}^{\prime}(b)\right]^{2} \\
& \times\left[\left(\omega_{r}-k_{/ /} v_{/ /}\right) \partial_{v_{\perp}}+k_{/ /} v_{\perp} \partial_{v_{/ /}}\right] f_{h}
\end{aligned}
$$

where

$$
\begin{aligned}
G \equiv & \omega_{r} \partial_{\omega_{r}} \Lambda_{r}=2\left[\frac{\omega_{p i}^{2} \omega_{r}^{2}}{\left(\Omega_{i}^{2}-\omega_{r}^{2}\right)^{2}} \sin ^{2} \phi+\frac{m_{i}}{m_{e}} \frac{\omega_{r}^{2}}{\Omega_{i}^{2}} \cos ^{2} \phi\right] N^{2} \\
& +2 \frac{m_{i}}{m_{e}} \frac{\omega_{p i}^{4}}{\left(\Omega_{i}^{2}-\omega_{r}^{2}\right)^{2}}\left[\left(2-\frac{m_{i}+m_{e}}{m_{i}} \sin ^{2} \phi\right) N^{2}-\frac{\omega_{p i}^{2}}{\omega_{r}^{2}}\right],
\end{aligned}
$$

$J_{s}$ are the usual Bessel functions of order $s$ and $J_{s}{ }^{\prime}$ are their corresponding first derivatives with respect to $b=k_{\perp} v_{\perp} / \Omega_{i}$.

Because $k_{/ /} v_{/ /} \ll \Omega_{i}$ and the presence of the Dirac-delta function, $\delta\left(\omega_{r}-s \Omega_{i}-k_{/ /} v_{/ /}\right)$, in eq. (11), the summation is reduced to the resonant term, $s=1$, that correlates to the fundamental frequency of the EMIC waves (i.e. $\omega_{r} \sim \Omega_{i}$ ). Consequently, using the combined distribution function of the suprathermal ions of eq. (1), the normalized growth rate can be written as

$$
\begin{aligned}
\frac{\gamma}{\Omega_{i}}= & \frac{n_{h}}{n_{0}} \frac{\omega_{p i}^{2}}{\omega_{r} k_{/ /} v_{T_{/ /}}} \frac{\sqrt{2 \pi}}{\alpha ! G}\left(\alpha I_{1}+\frac{A_{T}-\alpha}{\alpha+1} I_{2}-\frac{A_{T}+1}{\alpha+1} \frac{\omega_{r}}{\Omega_{i}} I_{2}\right) \\
& \times \exp \left[-\frac{\left(\Omega_{i}-\omega_{r}\right)^{2}}{2 k_{/ /}^{2} v_{T_{/ /}}^{2}}\right],
\end{aligned}
$$

where the integrals

$$
\begin{aligned}
I_{1}= & \int_{0}^{\infty} \mathrm{d} x x^{\alpha} \mathrm{e}^{-x}\left[\left(\lambda_{x y}+\frac{\Omega_{i}-\omega_{r}}{\Omega_{i}} \lambda_{y z} \tan ^{2} \phi\right) \frac{J_{1}\left(b_{0} \sqrt{x}\right)}{b_{0} \sqrt{x}}\right. \\
& \left.-\lambda_{y y} J_{1}^{\prime}\left(b_{0} \sqrt{x}\right)\right]^{2},
\end{aligned}
$$

and

$$
\begin{aligned}
I_{2}= & \int_{0}^{\infty} \mathrm{d} x x^{\alpha+1} \mathrm{e}^{-x}\left[\left(\lambda_{x y}+\frac{\Omega_{i}-\omega_{r}}{\Omega_{i}} \lambda_{y z} \tan ^{2} \phi\right) \frac{J_{1}\left(b_{0} \sqrt{x}\right)}{b_{0} \sqrt{x}}\right. \\
& \left.-\lambda_{y y} J_{1}^{\prime}\left(b_{0} \sqrt{x}\right)\right]^{2},
\end{aligned}
$$

and the parameters $x=v_{\perp}^{2} / 2 v_{T_{\perp}}^{2}$ and $b_{0}=\sqrt{2} k_{\perp} v_{T_{\perp}} / \Omega_{i}$.

\section{Results and discussion}

Figure 1(a) depicts the plot of the peak growth rate as a function of the propagation angle $\phi$, determined by varying the frequency $\omega_{r}$, for three different loss-cone indices, $\alpha=0,1$ and 3, with the effective anisotropic index taken to be $A_{T}=3$. The growth rate has been normalized by $\left(n_{0} / n_{h}\right) \Omega_{i}$ and the parameters $\varepsilon_{\perp}=20 \mathrm{keV}, m_{i} / m_{e}=1836$, and $\omega_{p i} / \Omega_{i}=40$ have been used in the numerical calculation.

From Figure 1(a), it is found that for the case when $\alpha=0$ (i.e. the temperature anisotropy alone contributes to the driven free energy of the suprathermal ions), the growth rate steadily decreases as the propagation angle $\phi$ varies between 0 (parallel propagation) and $\pi / 2$ (perpendicular propagation). The maximal growth rate occurs when $\phi=0$. However, for the cases when $\alpha=1$ and 3, after gently decreasing with $\phi$ in the regime of $\phi<1$, the growth rate then rapidly increases and reaches its maximum between $1.3<\phi<1.5$ (i.e. quasiperpendicular directions). Moreover, the maximal growth rate in the quasi-perpendicular direction is remarkably enhanced by larger loss-cone indices. This clearly indicates that the growth of quasi-perpendicular propagating EMIC waves is driven by the loss-cone distribution of the suprathermal ions.

Figure 1(b)-(d) show the variation in the growth rate with wave frequency $\omega_{r} / \Omega_{i}$ for $\alpha=0,1$ and 3 , respectively. A distinct characteristic in each of these plots is strong absorption at frequencies between $0.8 \Omega_{i}$ and $0.9 \Omega_{i}$. Also, a peak in the growth rate appears at frequencies near $0.7 \Omega_{i}$, except for the quasi-perpendicular propagating cases at $\phi \simeq 1.4$. These strong absorptions can be clearly attributed to the term that is proportional to the wave frequency $\omega_{r} / \Omega_{i}$ presented in eq. (13). For the quasi-perpendicular propagating case however, the EMIC wave continues to increase in this regime because of the loss-cone driven for $\alpha=1$ and 3, as shown in Figure 1(c) and (d). Figure 1(a) further shows that for quasi-parallel propagating EMIC waves in the region of $\phi<0.5$, the growth rate does not depend obviously on the loss-cone distribution, whereas for the quasi-perpendicular propagating case within $1.3<\phi<1.5$, the loss-cone distribution strongly affects the growth rate of EMIC waves. 

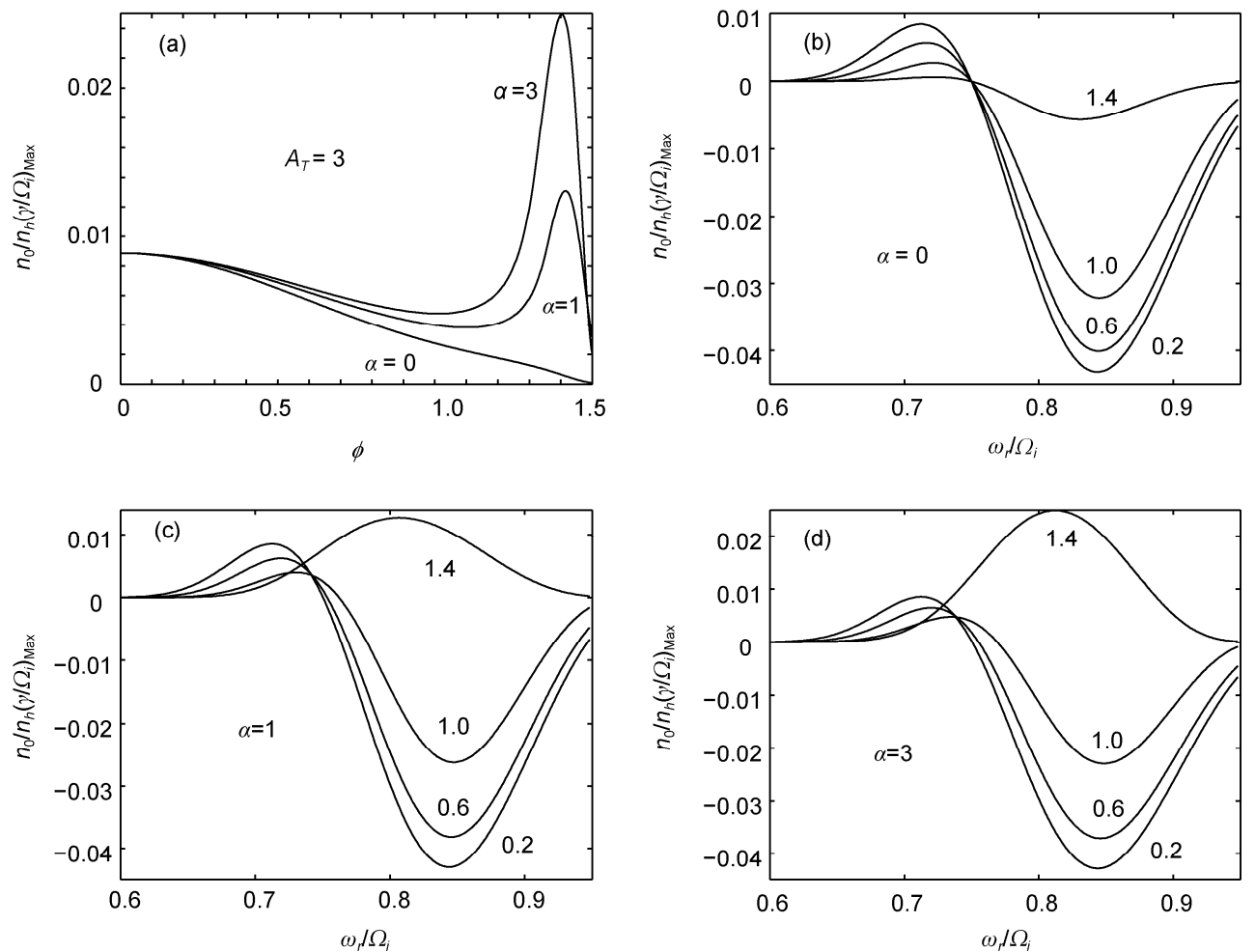

Figure 1 The normalized growth rate $\left(n_{0} / n_{h}\right) \gamma / \Omega_{h}$. (a) The peak growth rate versus the propagation angle $\phi$ for loss-cone indices $\alpha=0,1$ and 3, with anisotropic index taken to be $A_{T}=3$; (b)-(d) the variation in the growth rate with the normalized frequency $\omega_{r} / \Omega_{i}$ for the propagation angles $\phi=0.2,0.6,1.0$ and 1.4. Other parameters, $\varepsilon_{\perp}=20 \mathrm{keV}$, and $\omega_{p i} / \Omega_{i}=40$ have been used in the numerical calculation.

Figure 2(a) depicts the plot of the peak growth rate as a function of propagation angle $\phi$, determined by varying the frequency $\omega_{r}$, for three different anisotropic indices $A_{T}=1,3$ and 7, with loss-cone index taken to be $\alpha=3$. The other parameters have been taken to be the same as those in Figure 1.

From Figure 2(a), it is found that the maximal growth rate in the range $0<\phi<1$ is enhanced remarkably by larger anisotropic indices. In particular, for the case when the anisotropic indices are large, $A_{T}>\alpha$, for example, $A_{T}=7>\alpha=3$, as in Figure 2(a), the growth rate still decreases steadily when the propagation angle $\phi$ varies between 0 and $\pi / 2$. The maximal growth rate occurs for parallel propagating EMIC waves because, in this case, the temperature anisotropy dominates the wave's growth. However, for the quasiperpendicular propagating cases, in the rather narrow range of $1.3<\phi<1.5$, the wave growth again is dominated by the loss-cone distribution, as shown in Figure 2(a). In addition, Figure 2(a) shows that for smaller anisotropic indices, $A_{T} \leqslant \alpha$, the growth rate hardly decreases in of the region $\phi<1$ because of the effect of the loss-cone distribution.

Figure 2(b)-(d) show the variation of the growth rate with the wave frequency $\omega_{r} / \Omega_{i}$ for $A_{T}=7,3$ and 1 , respectively. These results are similar to those shown in Figure 1 (b)-(d). There is clearly strong absorption of the quasi- parallel propagating waves for $\phi \leqslant 1$ and a peak in the quasi-perpendicular propagating waves at $\phi \simeq 1.4$. One of the most important differences between Figure 1(b) and Figure 2 (b) is the peak in the growth rate evident in the latter case. This substantial growth can be attributed to the strongly anisotropic distribution with an anisotropic index $A_{T}>\alpha$, e.g. $A_{T}=7>\alpha=3$, which results in a remarkable enhancement of the peak growth rate as $\phi$ decreases. Furthermore, notice that this peak frequency drifts towards slightly lower frequencies as the propagation angle decreases.

\section{Conclusions}

Electromagnetic ion cyclotron (EMIC) waves are interesting because of their potential importance in the acceleration and heating of high-speed solar wind streams, as well as their potential links to the heating of heavy ions and ion diffusion in the magnetosphere. In addition, EMIC waves have potential significance in the ${ }^{3} \mathrm{He}$-rich events in impulsive flares in the solar corona. EMIC waves are often observed in various regions of the magnetosphere that can be excited by the ion temperature anisotropy or by the loss-cone distribution. However, in situ measurements by satellites in space plasmas have demonstrated that there are a large variety of microscopic velocity distributions of ions that often cannot be 

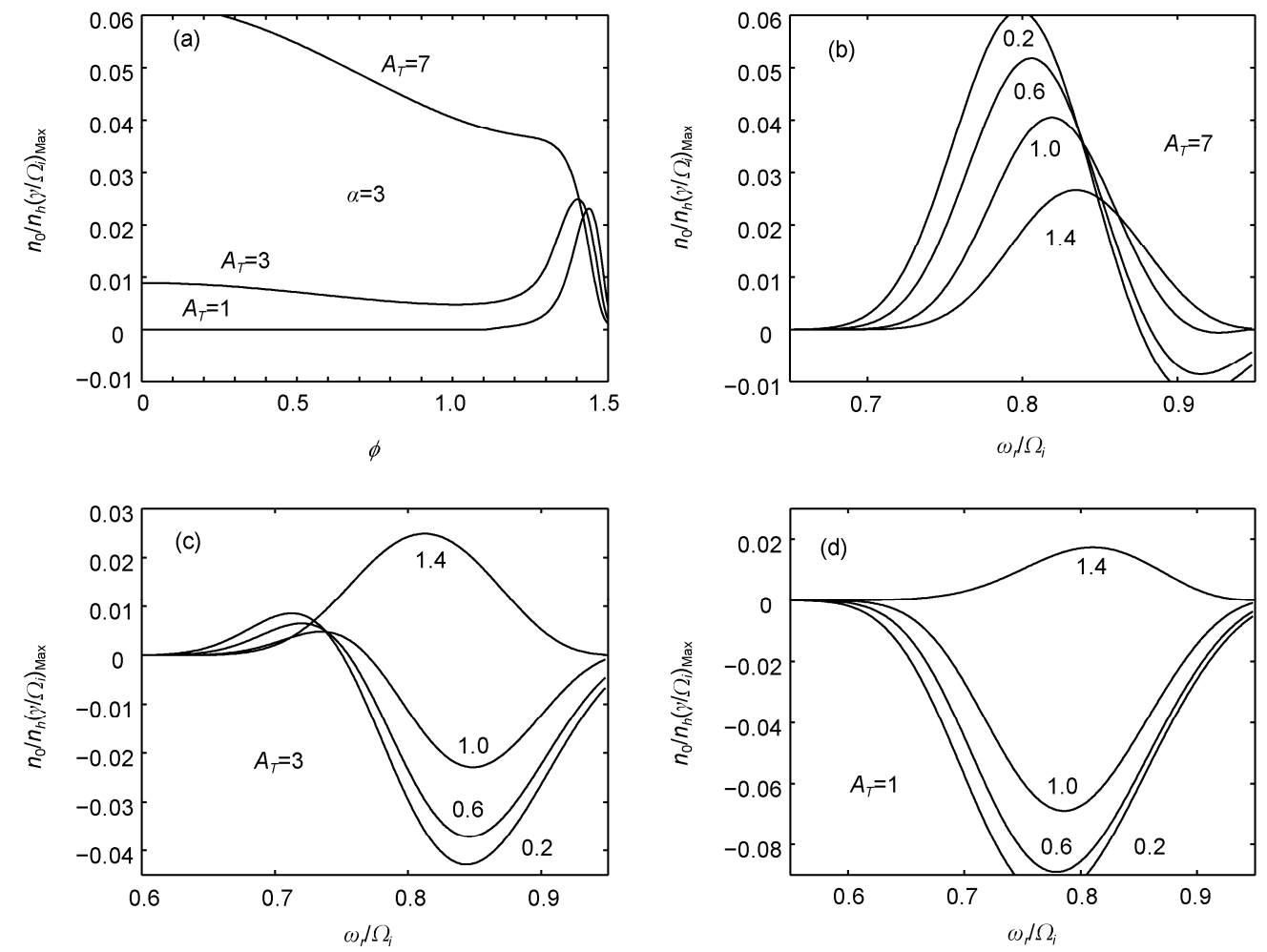

Figure 2 Normalized growth rate $\left(n_{0} / n_{h}\right) \gamma / \Omega_{i}$. (a) The peak growth rate versus the propagation angle $\phi$ for effective anisotropic indices $A_{T}=1,3$ and 7 , with loss-cone index taken to be $\alpha=3$; (b)-(d) the variation in the growth rate with the normalized frequency $\omega_{r} / \Omega$ for propagation angles $\phi=0.2,0.6,1.0$ and 1.4. Other parameters, $\varepsilon_{\perp}=20 \mathrm{keV}$, and $\omega_{p i} / \Omega_{i}=40$ have been used in the numerical calculation.

modeled by a simple distribution function.

In this paper, the parameter-dependence of EMIC instabilities is investigated with a combined loss-cone and temperature anisotropy distribution for suprathermal ions and a calculation of the linear growth rate of EMIC waves with an arbitrary propagation angle is presented. The results show that for a wide range of the propagation angle $0<\phi<1$, the growth rate decreases steadily with $\phi$, while the maximal growth, which occurs in the parallel direction $(\phi=0)$, increases remarkably with the anisotropic indices $A_{T}$. However, in the regime of $\phi>1$ the growth rate rapidly increases and reaches a "perpendicular" maximum between $1.3<\phi<$ 1.5 (i.e. quasi-perpendicular directions). Moreover, the "perpendicular" maximal growth rate is exceptionally enhanced by a larger loss-cone index $\alpha$.

The variation in the growth rate with the frequency $\omega_{r} / \Omega_{i}$ shows a strong absorption occurring at frequencies between $0.8 \Omega_{i}$ and $0.9 \Omega_{i}$ as well as a peak in the wave growth at frequencies near $0.7 \Omega$, with the exception being the quasi-perpendicular propagating cases at $\phi \simeq 1.4$, for which the EMIC wave increases because of the loss-cone driven for $\alpha \neq 0$. In fact, for quasi-parallel propagating EMIC waves with $\phi<0.5$, the growth rate shows little dependence on the loss-cone distribution, whereas for the quasi- perpendicular propagating case within $1.3<\phi<1.5$, the loss-cone distribution strongly affects the growth rate of EMIC waves.

These features imply that the free energy associated with the loss-cone distribution is primarily responsible for the excitation of perpendicular propagating EMIC waves, while the predominant contribution to the excitation of parallel propagating EMIC waves comes from the energy associated with the temperature anisotropy distribution. However, in situ satellite measurements have demonstrated that the actual microscopic velocity distributions of particles in space plasmas are characterized by a complex, multi-distribution combination. For instance, at high latitudes of the Earth's magnetosphere, the microscopic velocity distribution of ions usually consists of a combination of the loss-cone and the temperature anisotropy distributions. The results presented here show that perpendicular propagating EMIC waves can be driven efficiently by these complex distributions with combination characteristics, which gives a more comprehensive understanding of the excitation and generation mechanisms for EMIC waves in space plasmas.

This work was supported by the National Natural Science Foundation of China (10973043, 41074107) and National Basic Research Program of China (2011CB811402). 
1 Huang J G, Han J W, Li H W, et al. Mechanism and operation parameters of a plasma-driven micro-particle accelerator. Chinese Sci Bull, 2009, 54: 616-622

2 Li L Y, Cao J B, Zhou G C, et al. Shrinkage of magnetosphere observed by TC-1 satellite during the high-speed solar wind stream. Sci China Ser E-Tech Sci, 2008, 51: 1695-1703

3 Shen C, Liu Z X, Escoubet C P, et al. Surveys on magnetospheric plasmas based on the Double Star Project (DSP) exploration. Sci China Ser E-Tech Sci, 2008, 51: 1639-1647

4 Yue C, Zong Q G, Wang Y F. Response of the magneticfield and plasmas at the geosynchronous orbit to interplanetary shock. Chinese Sci Bull, 2009, 54: 4241-4252

5 Young D T, Perraut S, Roux R, et al. Wave-particle interactions near $\Omega_{\mathrm{He}+}$ observed on GEOS 1 and GEOS 2, Propagation of ion cyclotron waves in $\mathrm{He}^{+}$-rich plasma. J Geophys Res, 1981, 86: 6755-6772

6 Fraser B J. Observations of ion cyclotron waves near synchronous orbit and on the ground. Space Sci Rev, 1985, 42: 357-374

7 Erlandson R L, Zanetti L J, Potemra T A, et al. Viking magnetic and electricfield observation of Pc 1 waves at high latitudes. J Geophys Res, 1990, 95: 5941-5955

8 Anderson B J, Erlandson R E, Zanetti L J. A statistical study of Pc 1-2 magnetic pulsations in the equatorial magnetosphere. 1 Equatorial occurrence distributions. J Geophys Res, 1992, 97: 3075-3088

9 Anderson B J, Erlandson R E, Zanetti L J. A statistical study of Pc 1-2 magnetic pulsations in the equatorial magnetosphere. 2 Wave properties. J Geophys Res, 1992, 97: 3089-3101

10 Xiao F L, Tian T, Chen L X. Bounce-averaged pitch-angle diffusion by electromagnetic ion cyclotron waves in multi-ion plasmas. Chin Phys Lett, 2009, 26: 059402

11 Xiao F L, Tian T, Chen L G, et al. Evolution of ring current protons induced by electromagnetic ion cyclotron waves. Chin Phys Lett, 2009, 26: 119401

12 Hollweg J V. Transition region, corona, and solar wind in coronal holes. J Geophys Res, 1986, 91: 4111-4125

13 Hollweg J V, Isenberg P A. Generation of the fast solar wind: A review with emphasis on the resonant cyclotron interaction. J Geophys Res, 2002, 107: 1147

14 Cramer S R. Coronal holes and the high-speed solar wind. Space Sci Rev, 2002, 101: 229-294

15 Lu Q M, Wang S. Proton and $\mathrm{He}^{2+}$ temperature anisotropies in the solar wind driven by ion cyclotron waves. Chin J Astron Astropys, 2005, 5: 184-192

16 Lu Q M, Chen L. Ion heating by a spectrum of obliquely propagating low-frequency Alfven waves. Astrophys J, 2009, 704: 743-749

17 Mauk B H. Helium resonance and dispersion effects on geostationary Alven/ion cyclotron waves. J Geophys Res, 1982, 87: 91079119
18 Berchem J R, Gendrin R. Nonresonant interaction of heavy ions with electromagnetic ion cyclotron waves. J Geophys Res, 1985, 90: 10945-10960

19 Temerin M, Roth I. The production of ${ }^{3} \mathrm{He}$ and heavy ion enrichments in ${ }^{3} \mathrm{He}$-rich ares by electromagnetic hydrogen cyclotron waves. Astrophys J, 1992, 391: L105-L108

20 Miller J A, Vinas A F. Ion acceleration and abundance enhancements by electron beam instabilities in impulsive solar flares. Astrophys J, 1993, 412: 386-412

21 Gary S P, Anderson B J, Denton R E, et al. Ion anisotropies in the magnetosheath. Geophys Res Lett, 1993, 20: 1767-1770

22 Anderson B J, Fuselier S A, Gary S P, et al. Magnetic spectral signatures in the Earth's magnetosheath and plasma depletion layer. J Geophys Res, 1994, 99: 5877-5891

23 Gray S P. Theory of Space Plasma Microinstabilities. New York: Cambridge University Press, 1993

24 Gray S P, McKean M E, Winske D. Ion cyclotron anisotropy instabilities in the magnetosheath: Theory and simulations. J Geophys Res, 1993, 98: 3936-3971

25 Gray S P, Lee A. The ion cyclotron anisotropy instability and the inverse correlation between proton anisotropy and proton beta. J Geophys Res, 1994, 99: 11297-11301

26 Gray S P, Wang J, Winske D, et al. Proton temperature anisotropy upper bound. J Geophys Res, 1997, 102: 27159-27169

27 Lu Q M, Guo F, Wang S. Magnetic spectral signatures in the terrestrial plasma depletion layer: Hybrid simulations. J Geophys Res, 2006, 111: A04207

28 Ludlow G R. Growth of obliquely propagating ion cyclotron waves in the magnetosphere. J Geophys Res, 1989, 94: 15385-15391

29 Denton R E, Hudson M K, Roth I. Loss-cone-driven ion cyclotron waves in the magnetosphere. J Geophys Res, 1992, 97: 12093-12103

30 Xue S, Thorne R M, Summers D. Growth and damping of oblique electromagnetic ion cyclotron waves in the Earth's magnetosphere. J Geophys Res, 1996, 101: 15457-15466

31 Xiao F L, Zhou Q H, He H Y, et al. Proton cyclotron instability threshold condition of suprathermal protons by kappa distribution. Plasma Sci Tech, 2007, 9: 545-549

32 Gray S P, Vazquez V M, Winske D E. Electromagnetic proton cyclotron instability: Proton velocity distributions. J Geophys Res, 1996, 101: $13327-13333$

33 Melrose D B. Plasma Astrophysics. New York: Gardon and Breach, 1980. 51-53

34 Stix T H. Waves in Plasmas. New York: American Institute of Physics, 1992. 247-262

35 Wu C S. Kinetic cyclotron and synchrotron maser instabilities: Radio emission processes by direct amplifcation of radiation. Space Sci Rev, 1985, 41: 215-298

Open Access This article is distributed under the terms of the Creative Commons Attribution License which permits any use, distribution, and reproduction in any medium, provided the original author(s) and source are credited. 